\title{
Política Monetaria en el Ecuador.
}

\section{Monetary Policy in Ecuador.}

Lenin Agustín Chamba Bastidas. ${ }^{1}$, Doris Maribel Sánchez Lunavictoria. ${ }^{2}$, Homero Eudoro Suárez Navarrete. ${ }^{3}$, María Alexandra Procel Silva. ${ }^{4}$

DOI: https://doi.org/10.33262/visionariodigital.v3i2.476

\section{Resumen.}

Con la promulgación de la Ley General de Instituciones del Sector Financiero en 1994, se facilita la creación de una institución financiera y reduce los sistemas de control, de 24 bancos que había en el año 1994, aumentaron a 96 en 1999, denota con claridad el facilismo que se dio desde el punto de vista legal para que se puedan crear bancos en el Ecuador a efectos de resolver los problemas económicos del país, es necesario ejecutar una política de manera integral. Se debe considerar la política del sector externo, especialmente de los países con los cuales tenemos mayor relación comercial o de aquellos que tienen mucha influencia a nivel mundial, la realidad económica es diferente y por lo tanto los efectos y acciones posiblemente difieran entre sí.

Palabras clave: Política Monetaria, Ley General, Instituciones Financieras, Bancos

\begin{abstract}
With the enactment of the General Law of Financial Sector Institutions in 1994, it facilitates the creation of a financial institution and reduces the control systems, from 24 banks that were in 1994, increased to 96 in 1999, clearly denotes the Facility that was given from the legal point of view so that banks can be created in Ecuador in order to solve the economic problems of the country, it is necessary to implement a policy in a comprehensive manner. The policy of the external sector must be considered, especially

\footnotetext{
${ }^{1}$ Facultad de Administración de Empresas, Escuela Superior Politécnica de Chimborazo, lenin.chamba@espoch.edu.ec

${ }^{2}$ Facultad de Administración de Empresas, Escuela Superior Politécnica de Chimborazo, doli.sanchez@espoch.edu.ec

${ }^{3}$ Facultad de Administración de Empresas, Escuela Superior Politécnica de Chimborazo, homero.suarez@espoch.edu.ec

${ }^{4}$ Facultad de Administración de Empresas, Escuela Superior Politécnica de Chimborazo, maria.procel@espoch.edu.ec
} 
of the countries with which we have the greatest commercial relationship or those that have a great influence at world level, the economic reality is different and therefore the effects and actions may differ among themselves.

Keywords: Monetary Policy, General Law, Financial Institutions, Banks

\section{Introducción.}

Al igual que la política fiscal, la política monetaria ${ }^{5}$ también es una rama de la política económica y se la utiliza como herramienta para influir de manera positiva en la economía de un determinado país.

Para la mayoría de las personas, la crisis financiera de 1999, la más alta del período en el Ecuador y la causante de muchos problemas económicos y sociales, nace aparentemente de una relación de tipo de cambio, si bien es lo que se veía en aquellos días, la distancia del dólar y la moneda ecuatoriana de manera acelerada, incrementando el índice de pobreza en la mayoría de ecuatorianos y en otros casos, mejorando sustancialmente el patrimonio o al menos aquel que pudo obtener dólares, no tuvo afectación significativa.

Con la promulgación de la Ley General de Instituciones del Sector Financiero en 1994, se facilita la creación de una institución financiera y reduce los sistemas de control, evento que en la actualidad es en parte similar con lo que respecta al sector financiero cooperativo, sector en el que la Súper Intendencia de Economía Popular y Solidaria ha intervenido con frecuencia debido a los problemas estructurales que presentan ciertas cooperativas.

De 24 bancos que habían en el año 1994, aumentaron a 96 en 1999, dato que denota con claridad el facilismo que se dio desde el punto de vista legal para que se puedan crear bancos en el Ecuador y sin el control representativo, a tal punto que los créditos vinculados a sus propios accionistas o administradores sin las suficientes garantías aparentemente, no se podían recuperar por algún medio y con la finalidad de evitar la crisis y garantizar el sistema financiero, es el Estado quien tiene que aportar los recursos, muchos de ellos de las reservas, con el firme propósito de salvar el sistema financiero,

\footnotetext{
${ }^{5}$ Acción de las autoridades monetarias dirigida a controlar las variaciones en cantidad disponible o en el coste del dinero en el mercado con la finalidad de conseguir determinados objetivos de carácter macroeconómico. Estos objetivos son generalmente el control de la inflación, la reducción del desempleo, la consecución de una tasa de crecimiento de la renta o producción de una tasa de crecimiento de la renta o producción real y la mejora en el saldo de la balanza de pagos. Los principales instrumentos con los que cuenta para alcanzar dichos objetivos son el coeficiente de caja y las operaciones de redescuento, de crédito y de mercado abierto.
} 
entonces claro, la estrategia fue interesante, quebrar el banco y esperar que el estado lo salve, sin importar el nivel de afectación y efectos negativos al Estado y el pueblo en general.

Si a lo indicado le sumamos otros fenómenos de otro sector, pero que de igual manera agudizan el problema económico, como la guerra con el Perú en 1995, los terremotos que paralizaron la explotación y exportación de nuestro producto preciado, el petróleo, los bajos precios del petróleo y el fenómeno climático denominado El Niño, hicieron que la política monetaria sea tan efímera para solucionar problema de tal dimensión.

La capacidad u oferta de servicios bancarios era superior a la demanda, motivo por el cual los precios del recurso dinero o las tasas de interés como comúnmente se las conoce, tendieron a modificarse por tratar de captar el ahorro y como era de esperarse, la especulación por obtener dólares y llevarlos al exterior fue mayor que la atención de las tasas bancarias.

Cada decisión que se tomaba después del año 1994, simplemente no era suficiente para solucionar el problema presentado; es muy probable que la convertibilidad que proponía el ex presidente Abdala Bucarán, pudo ser un freno al tipo de cambio e inflación y al problema económico que en general se veía venir, sin embargo, los problemas políticos y de corrupción que se manifiesta en su momento, promovieron una gran resistencia por parte de la ciudadanía, dejando sin la posibilidad que se ejecute el planteamiento de convertibilidad. ${ }^{6}$

La dolarización que para unos no fue la solución, considero que, si evitó gran parte del empeoramiento del problema, si en un mediano plazo el Ecuador presentara problemas económicos, pienso que no serían por causa de la dolarización, sino por causa de la producción, recordemos que el sector real es el dirimente en el camino de la economía de un país y es justamente el petróleo, el que está ya comprometido.

\section{Desarrollo}

\footnotetext{
${ }^{6}$ La convertibilidad es un sistema monetario que fija el valor de una moneda con el de otra considerada más estable (generalmente el dólar o el euro) u otro patrón (por ejemplo el oro). La ventaja de un sistema monetario convertible, es lograr previsibilidad en el valor de una moneda y evitar saltos inesperados en su cotización. La desventaja es la rigidez monetaria, que puede llegar a causar valores irreales (sobrevaloración o subvaloración), impidiendo que la elasticidad del precio de la moneda corrija este tipo de situaciones.
} 
Como bien se observa en la definición de la política monetaria, ésta será trabajada desde el análisis de la variación del PIB, inflación, desempleo y balanza de pagos.

La teoría de Keynes fué y es una de las más representativas en lo que respecta a política monetaria, sin dejar de lado el fundamental aporte de los monetaristas como Milton Friedman y la escuela de Chicago, quién tomando las sugerencias de otros autores, dio batalla significativa al pensamiento de Keynes, consideró que el problema de la inflación se resolvería con restricciones de la oferta monetaria y una tasa constante y moderada.

Sin embargo, Keynes planteó su teoría basada en que la demanda global que es la que mueve la actividad económica y para resolver el problema de desempleo con la política monetaria, propone bajar las tasas de interés para que los inversionistas tengan recursos más baratos para trabajar y así fomentar las exportaciones mediante una disminución del tipo de cambio que permita combatir el desempleo.

Partimos de la siguiente ecuación.

$$
D_{g}=C+I+G+E_{X}
$$

$$
\text { Desempleo } \Uparrow D_{g}=\Downarrow I+E_{X} \Uparrow \text { del tipo de cambio }
$$

Para combatir la inflación, básicamente es lo contrario de lo indicado en el párrafo anterior, esto es desestimular la demanda global, mediante la subida de las tasas de interés y promoviendo una elevación del tipo de cambio.

$$
\text { Inflación } \Downarrow D_{g}=\Uparrow I+E_{X} \Uparrow \text { del tipo de cambio }
$$

La teoría de Keynes como lo hemos venido revisando, está ligada a la demanda global mientras que la teoría de Milton Friedman está ligada a lo que es la oferta y de manera particular la oferta monetaria.

Según Michael Parkin y Eduardo Loria Díaz, los objetivos de la Política Monetaria son: máximo empleo, precios estables y tasas de interés a largo plazo moderadas. Estos 
objetivos podrían entrar en conflicto a mediano plazo y a largo plazo, podrían estar en armonía. (Loria, 2010)

Sin duda alguna, alcanzar estos y otros objetivos macroeconómicos, no es fácil a pesar que ciertos países cuentan con mejores condiciones, una de las causas principales que considero está discurrida con un eje transversal en cualquier tema económico, es la política internacional, las políticas que toman el sector externo independientemente de lo que se resuelva de manera interna, es fundamental considerar o al menos tener una base y seguimiento de lo que pretenden hacer otros países que tiene influencia directa con el nuestro.

\section{Gráfico 1}

Variación del PIB a precios constantes $(1990$ - 2016)

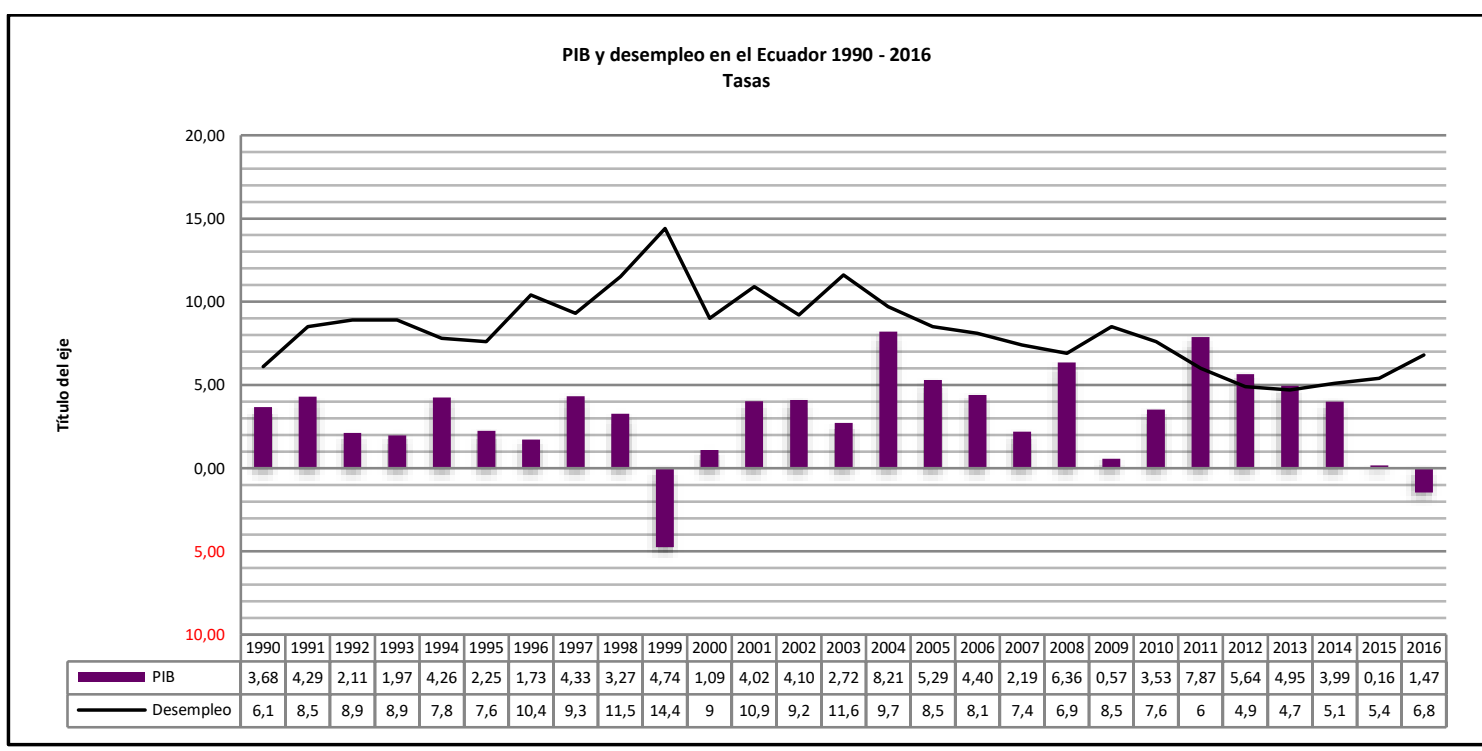

Fuente : CEPAL (https://www.cepal.org/es)

\section{Elaborado : Autor}

Si observamos el gráfico uno, podemos evidenciar con claridad la relación inversa que tuvo el PIB y el desempleo en el año 1999, mientras la inflación sube de manera excesiva, el PIB baja con velocidad y distancia similar.

Las tasas de interés posterior a la crisis, especialmente la tasa activa, sigue alta para el año 2001 (www.cepal.org, 2018) con relación al resto del periodo (tasa de 16,03) llegando hasta el 8,4 para el año 2016, mientras que la tasa pasiva esta relativamente en todo el periodo en un rango de 4 a 8 puntos. 
La distancia entre la tasa activa y pasiva entre el 2000 y 2004, es mayor que la del 2005 al 2016, lo que significa que saliendo de una crisis financiera significativa de 1999, la propuesta de alzar las tasas para disminuir la demanda global y así estabilizar el empleo y la inflación, posiblemente dieron un resultado positivo, ya que la tendencia de la curva tanto en inflación como desempleo, tendieron a la baja. Ahora bien, después de tan elevada curva en 1999 para ambos problemas, el escenario consolador, era que no suba más y se mantenga la curva, en este caso cambió la tendencia con pendiente negativa de las curvas, lo cual es de mayor beneficio.

\section{Gráfico 2}

Margen de interés neto (1996 - 2015)

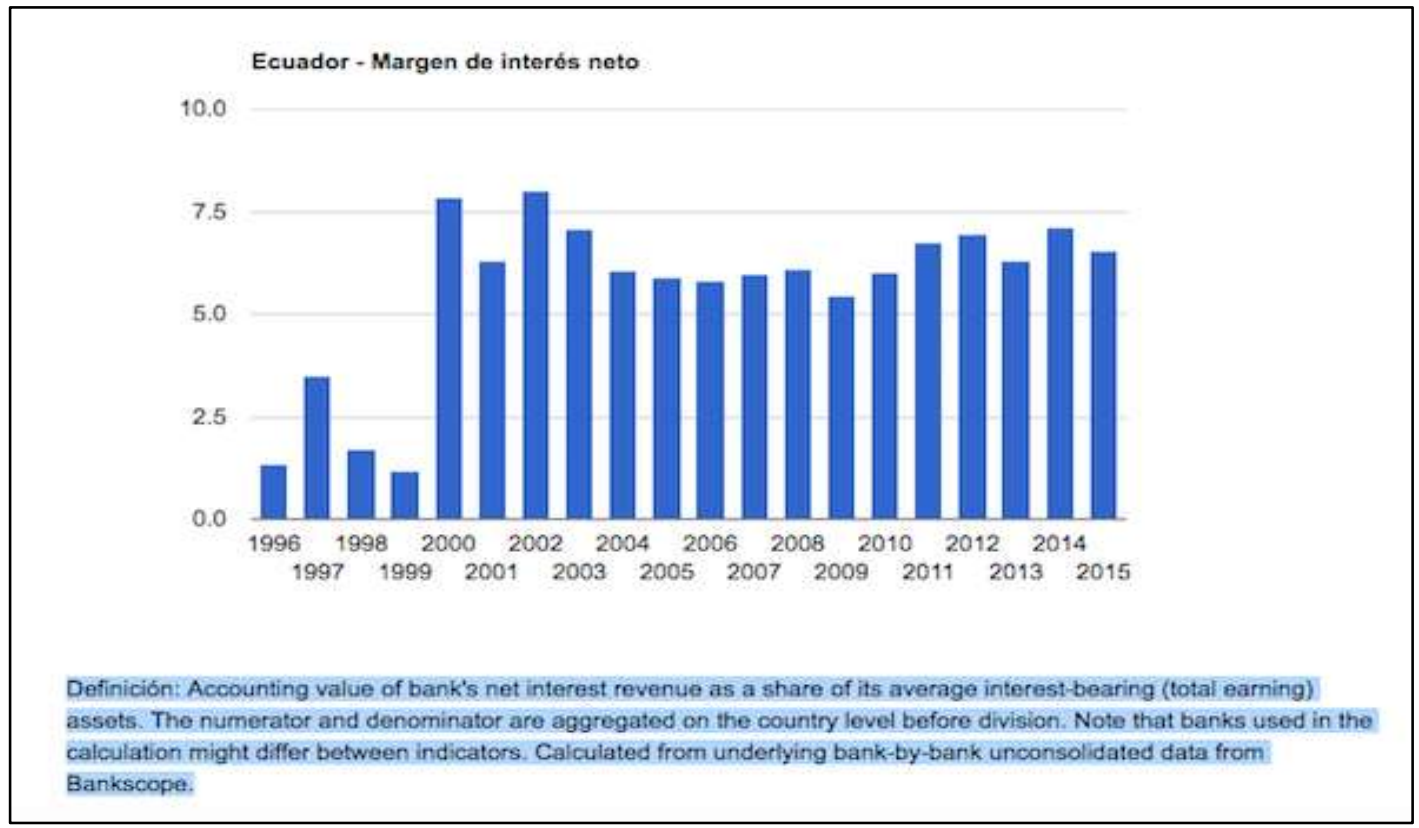

Fuente : CEPAL - (theglobaleconomy.com, 2008)

\section{Elaborado : Autor}

Respecto del gráfico dos, podemos denotar que en el año 1999, es cuando menos ingresos recibe el sector bancario, lo cual corrobora lo anteriormente indicado, el problema nace con las facilidades y falta de control para incrementar instituciones financieras y efectuar créditos vinculados, lo cual produce principalmente que la oferta de servicios es demasiado mayor para el mercado y por lo tanto los costos son muy altos, más el no pago de intereses ni capital por el tema de créditos vinculados, el resultado es bastante negativo. 
En el año 2000, se reactiva la economía y la curva de la tasa de crecimiento del PIB, comienza a tener pendiente positiva, efecto que obedece en gran parte al tema de dolarización. $^{7}$

También de puede observar que, la tasa del año 2015, es menor que la del año 2014 y la tendencia se deja ver para bajo es decir los ingresos netos van disminuyente en tendencia.

\section{Gráfico 3}

\section{Tasa de desempleo e inflación}
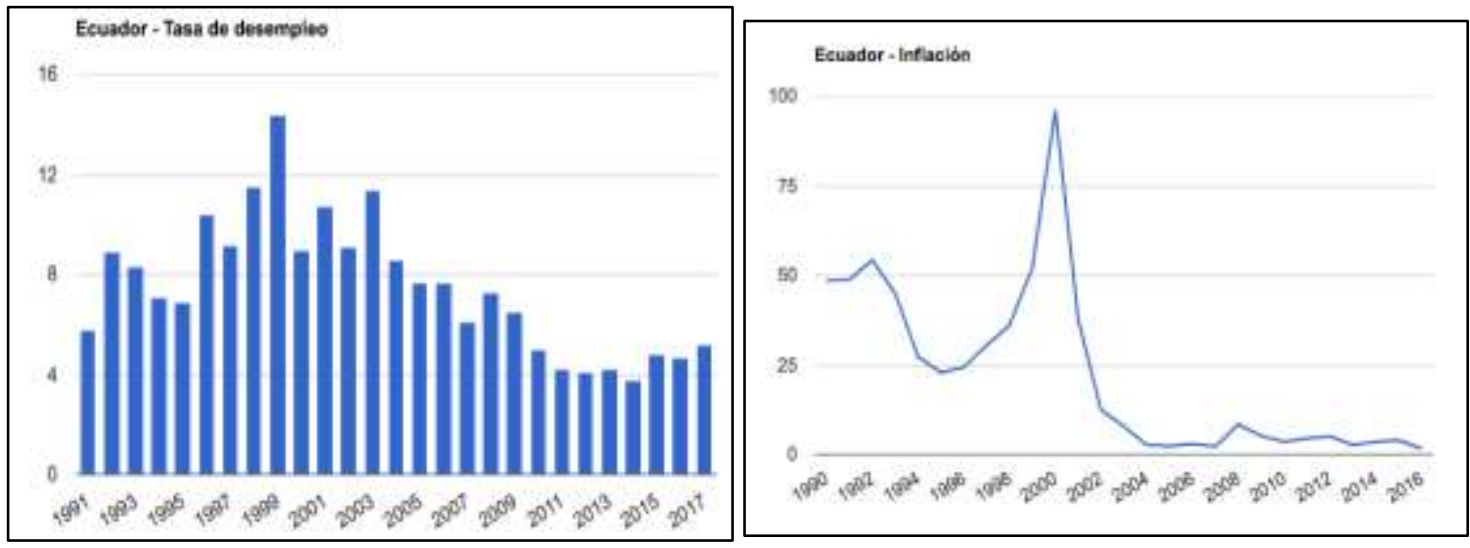

Fuente : Theglobaleconomy (theglobaleconomy.com, 2008)

\section{Elaborado : Autor}

La teoría explica que hay una relación inversa entre la inflación y el desempleo, cuando el uno sube el otro baja y viceversa, pero en las siguientes gráficas podeos confirmar en el Ecuador y para el año 1999 especialmente, crecieron las dos variables, la inflación y el desempleo.

Actualmente el Ecuador no cuenta con ciertos instrumentos que le permitan trabajar en política monetaria, como es el tipo de cambio, al no tener una moneda local, no se puede influir en bajar o subir las exportaciones.

\footnotetext{
${ }^{7}$ La dolarización es el proceso por el cual un país adopta de manera oficial el uso de la moneda estadounidense, de allí el origen del término, para su uso en transacciones económicas domésticas. La moneda extranjera estadounidense reemplaza a la moneda doméstica en todas sus funciones (reserva de valor, unidad de cuenta, medio de pago). Es adoptada como moneda de curso legal exclusiva o predominante, perdiendo o cediendo parte de soberanía monetaria.
} 
Las políticas restrictivas y expansivas deben ser ejecutadas con precisión y cautela, a diferencia de otras ciencias, la Economía no tiene un laboratorio que le permita hacer pruebas efectivas.

\section{Conclusiones.}

- A efectos de resolver los problemas económicos de un determinado país, es necesario ejecutar una política de manera integral, especulando todos los posibles escenarios que se pudieran presentar e involucrando cada herramienta económica a una alternativa global.

- Se debe considerar la política del sector externo, especialmente de los países con los cuales tenemos mayor relación comercial o de aquellos que tienen mucha influencia a nivel mundial.

- Las leyes que se promulgue, deben ser apegadas a hechos reales y objetivos, no se debe presumir lo que podría suceder, se debe hacer al menos un análisis que si bien no exime de riesgo alguna, pero si lo minimiza.

- Las teorías no se deben aplicar de manera estricta, ya que la realidad económica es diferente y por lo tanto los efectos a mismas acciones posiblemente difieran entre si.

\section{Referencias Bibliográficas.}

Constitución de la República de Ecuador. (2008). Quito: Registro Oficial .

Wikipedia. (09 de 02 de 2008).

https://es.wikipedia.org/wiki/Pol\%C3\%ADtica_fiscal. Recuperado el 09 de 02 de 2008, de Wikipedia: https://es.wikipedia.org/wiki/Pol\%C3\%ADtica_fiscal

Arthur Andersen . (1999). Diccionario de Economía y Negocios . Madrid, España: Espasa Calpe, S. A.

Roura, J. R. (2010). Politica Económica. (C. Sanchez, Ed.) Madrid, España: INTERAMERICANA DE ESPAÑA.

https://www.cepal.org/es. (s.f.). https://www.cepal.org/es. Recuperado el 05 de 01 de 2008, de http://interwp.cepal.org/cepalstat/engine/index.html:

http://interwp.cepal.org/cepalstat/engine/index.html

www.inec.gob.ec. (10 de 02 de 2008). Obtenido de http://www.ecuadorencifras.gob.ec/base-de-datos-censo-de-poblacion-y-vivienda/\#: http://www.ecuadorencifras.gob.ec/base-de-datos-censo-de-poblacion-y-vivienda/\#

https://www.iadb.org. (15 de 02 de 2008). https://www.iadb.org. Obtenido de https://publications.iadb.org/handle/11319/8601:

https://publications.iadb.org/handle/11319/8601 
ISSN: 2602-8506

theglobaleconomy.com. (03 de 01 de 2008). theglobaleconomy.com. Obtenido de http://es.theglobaleconomy.com/Ecuador/Government_size/:

http://es.theglobaleconomy.com/Ecuador/Government_size/

www.cepal.org. (16 de 02 de 2018). www.cepal.org. Obtenido de http://interwp.cepal.org/cepalstat/engine/index.html:

http://interwp.cepal.org/cepalstat/engine/index.html

Loria, M. P. (2010). Macroeconomía versión para Latinoamérica. (Vol. 9).

México DF, México: PEARSON. 
Para citar el artículo indexado.

Chamba L., Sánchez D., Suárez H., Procel M.. (2019). Política Monetaria en el Ecuador.. Revista electrónica Visionario Digital 3(2), 284-293. Recuperado desde: http://www.visionariodigital.org/

\section{Ciencia
Digital
\&uturaul}

El artículo que se publica es de exclusiva responsabilidad de los autores y no necesariamente reflejan el pensamiento de la Revista Ciencia Digital.

El articulo queda en propiedad de la revista y, por tanto, su publicación parcial y/o total en otro medio tiene que ser autorizado por el director de la Revista Ciencia Digital. 